\title{
ANALYSIS OF TIME-VARYING SYNCHRONIZATION OF EEG DURING SENTENCES IDENTIFICATION
}

\author{
Minfen Shen $^{1.2} \quad$ K. H. Ting ${ }^{2} \quad$ P. J. Beadle ${ }^{3} \quad$ F. H. Y. Chan ${ }^{2}$ \\ Science Research Centre, Shantou University, GD 515063, China. Email: mfshen@stu.edu.cn \\ ${ }^{2}$ The Centre of Biomedical Engineering, The University of Hong Kong, Hong Kong, China \\ ${ }^{3}$ The Scholl of System Engineering, The University of Portsmouth, Portsmouth, United Kingdom.
}

\begin{abstract}
The study of the synchronization of EEG signals can help us to understand the underlying cognitive processes and detect the learning deficiencies since the oscillatory states in the EEG reveal the rhythmic synchronous activity in large networks of neurons. As the changes of the physiological states and the relative environment exist when cognitive and information processing take place in different brain regions at different time, the practical EEGs therefore turn out to be extremely non-stationary processes. To investigate how these distributed brain regions are linked together and the information is exchanged with time, this paper proposes a modern time-frequency coherent analysis method that employs an alternative way for quantifying synchronization with both temporal and spacial resolution. Wavelet coherent spectrum is defined such that the degree of synchronization and information flow between different brain regions can be described. Several real EEG data are analysed under the cognitive tasks of sentences identification in both English and Chinese. The time-varying synchronization between the brain regions involved in the processing of sentences exhibited that a common neural network is activated by both English and Chinese sentences. The results of the presented method are helpful for studying the English and Chinese learning for the Chinese students.
\end{abstract}

Keywords: Neural signal processing, time-varying synchronization, sentences learning

\section{INTRODUCTION}

Recent research has shown that the brain works as a distributed parallel processing system. Assessment of the human brain functioning is frequently carried out by processing the brain electrical signals. Electroencephalogram (EEG) is regarded as the summed electrical activity of very large numbers of neurons. When the learning task is carried out, the corresponding cognitive and information processing take place in different brain regions. The investigation of the neuron synchronization of EEG signals enables to understand the underlying cognitive processes. The coherent analysis was often employed to measure the synchronization and the information flowing between two channel EEGs for the purpose of studying how these distributed brain regions are linked together and the corresponding information is exchanged. Traditional linear method for calculating the correlation between two time series in the frequency domain, coherence analysis, is valid for the stationary EEGs. There had existed some results showing that the coherence of EEG signals from different recording sites was often closely correlated with the cognitive processes [1-3].

However, most practical EEG signals often turns to be an extremely time-varying process [4-7]. The situation is more complicated since the state of the particular subsystem can change rapidly on a time scale of milliseconds. Thus, Investigation of the temporal and spacial interaction among the subsystems of the brain is needed to fully understand the neural information processing of the brain. The generic coherent analysis limits the dynamic synchrony analysis of the practical EEG signals [8-9]. Transient Fourier spectrum is often used to represent the physical quantity of non-stationary EEG signals. The short-time Fourier transform (STFT) may be interpreted as a Fourier spectrum of data that performs through a sliding window of finite extension. Different time and frequency set up the desired time-frequency representation. The significant drawback of this method was lack of temporal resolution that limits the application to the transient synchronization analysis of EEG recordings.

To investigate the synchronization of the EEGs when the subjects were identifying the specified sentences, a novel method for time-varying synchronous analysis via wavelet transform is developed in this paper. This alternative for quantifying synchronization with both temporal and spacial resolution enables to indicate the degrees of synchronization at each time. Cross wavelet spectrum is defined such that the cross wavelet magnitude spectra serves the coherent degree and the cross wavelet phase reveals the direction of the information flow between any two channels EEGs on different brain regions.

The paper is organized as the following. In section II, we describe the wavelet transform and define the wavelet coherence. Some simulation results are also discussed. In section III, the time-varying synchronization is applied to the real EEG data under the specified cognitive tasks. The 
preliminary experimental results are also discussed. Finally, the conclusion is addressed in section IV.

\section{TIME-VARYING SYNCHRONIZATION}

Our method combines the wavelet transform with the coherence to form a novel time-varying synchronization analysis that explores the amount of synchrony among the multiple channel brain activities.

Wavelet analysis has already been a very popular method for studying the non-stationary signal, whereas cross wavelet analysis can provide the degree of synchronization among the multiple channel EEGs. Morlet wavelet is chosen so that we can benefits from its nature of complex wavelet that provides not only the amplitude information, but also the phase relationship between any two EEG time series.

The continuous wavelet transform of the EEG time series is given by

$$
X(\tau, a)=|a|^{-1 / 2} \int_{-\infty}^{\infty} x(t) \varphi\left(\frac{t-\tau}{a}\right) d t
$$

Where the wavelet function is expressed as

$$
\varphi(t)=e^{-t^{2} / 2} e^{j w_{0} t}
$$

$X(\tau, a)$ denotes the wavelet transform of the given signal $x(t)$. The cross wavelet spectra between signal $i$ and $j$ is defined as

$$
\begin{gathered}
X_{i j}(\tau, a)=X_{i}(\tau, a) X_{j}^{*}(\tau, a) \\
=\left|X_{i j}(\tau, a)\right| \phi(\tau, a)
\end{gathered}
$$

The cross wavelet magnitude spectra reveal the degree of coherence between two signals, whereas the phase relationship represents the direction of the information flow of the transient brain activities.

To test the behavior of the presented method, two test processes with $1000 \mathrm{~ms}$ length were generated as:

$$
\begin{aligned}
& x_{1}(t)=\left\{\begin{array}{clc}
\sin (10 \pi t+30 \pi / 180) & \text { for } & 0 \leq t<250 \mathrm{~ms} \\
\sin (70 \pi t) & \text { for } & 250 \leq t<500 \mathrm{~ms} \\
\sin (40 \pi t-70 \pi / 180) & \text { for } & 500 \leq t<750 \mathrm{~ms} \\
\sin (24 \pi t+100 \pi / 180) & \text { for } & 750 \leq t<1000 \mathrm{~ms}
\end{array}\right. \\
& x_{2}(t)=\left\{\begin{array}{clc}
\sin (10 \pi t+30 \pi / 180) & \text { for } & 0 \leq t<250 \mathrm{~ms} \\
\sin (70 \pi t-100 \pi / 180) & \text { for } & 250 \leq t<500 \mathrm{~ms} \\
\sin (40 \pi t) & \text { for } & 500 \leq t<750 \mathrm{~ms} \\
\sin (24 \pi t+30 \pi / 180) & \text { for } & 750 \leq t<1000 \mathrm{~ms}
\end{array}\right.
\end{aligned}
$$
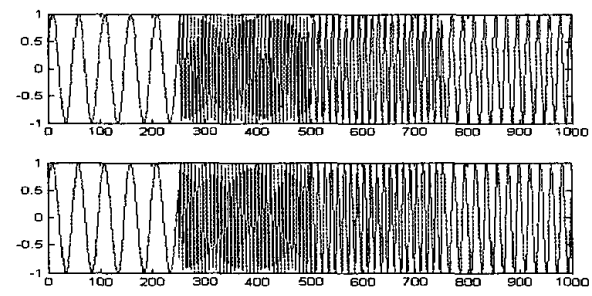

Fig. 1 Two test signals having 4 segments with the same frequency in each segment, but the distinguishing phase difference in segments 2,3 and 4 .

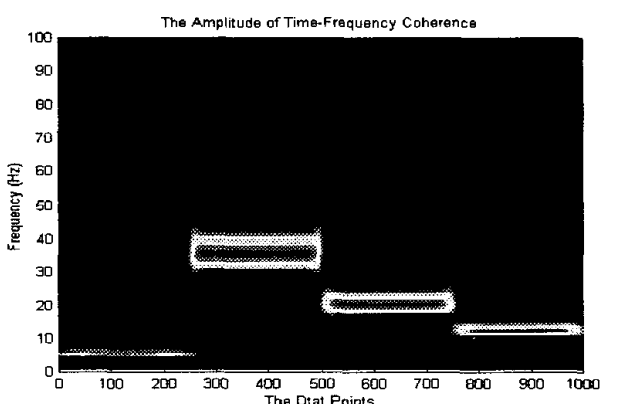

Fig. 2. The time-varying synchronization of the underlying signals is clearly identified.

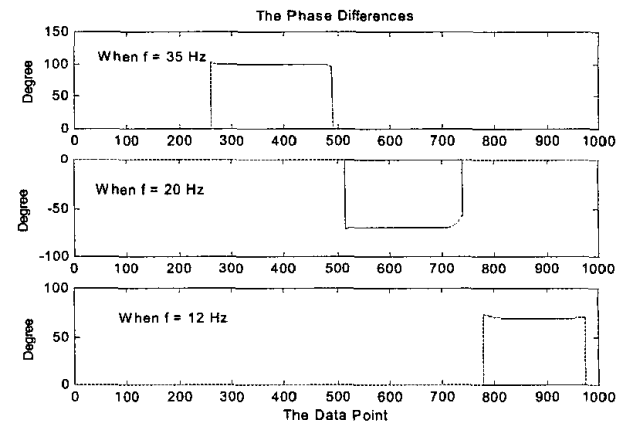

Fig. 3. The phase relationship, from top to bottom: the phase differences for segment 2,3 and 4 .

Fig. 1 shows two time-varying signals having 4 segments with different synchronization and phase relations in each segment. The mono-frequency is $5 \mathrm{~Hz}$ in segment 1 , $35 \mathrm{~Hz}$ in segment $2,20 \mathrm{~Hz}$ in segment 3 and $12 \mathrm{~Hz}$ in segment 4. Fig. 2 demonstrates the time-frequency coherence with frequency ranging from 1 to $100 \mathrm{~Hz}$ and time scale from 1 to $1000 \mathrm{~ms}$. The time-varying coherent structures of the underlying signals were clearly identified. The phase differences for segment 2,3 and 4 were also clearly estimated in Fig. 3 that is consistent to the maths representations. 


\section{Geodesic Sensor Net \\ 128 Channel V2.0}

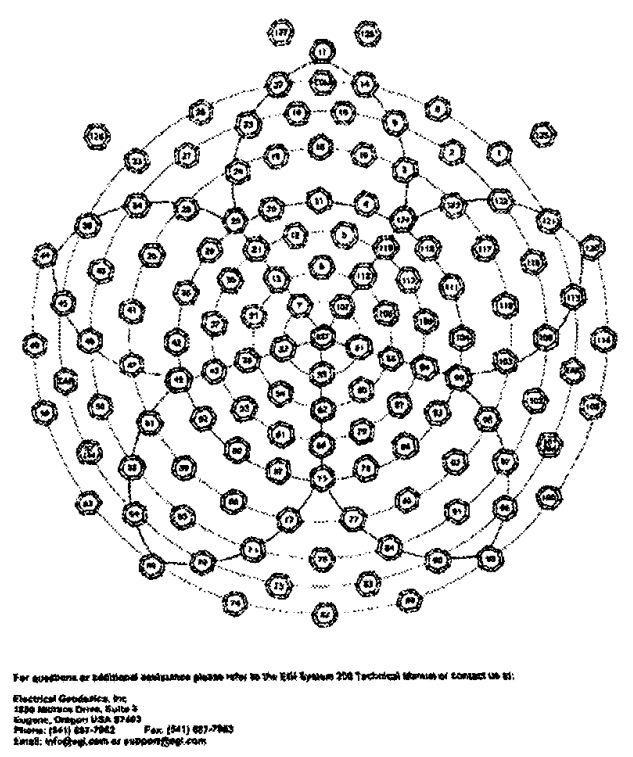

Fig. 4. 128-channel EEGs were collected via the Multi-dimensional Geodesic EEG/ERP system.

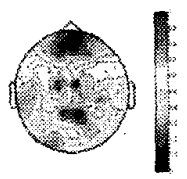

(a)

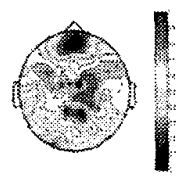

(b)

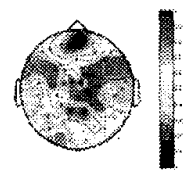

(c)
Fig.5. The topograph of dynamic synchronization when subjects identified the Chinese sentences. (a) Before identifying the sentence, the first periods of $1-25 \mathrm{~ms}$. (b) The result during the second periods of $26-150 \mathrm{~ms}$. (c) The result during the final periods of $151-250 \mathrm{~ms}$.

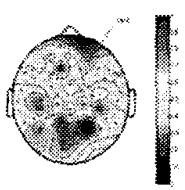

(a)

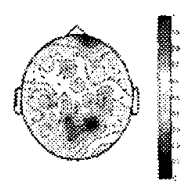

(b)

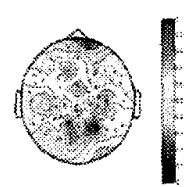

(c)
Fig. 6. The topographical mapping of dynamic synchronization when subjects identified the English sentences with three same periods as Fig.5.

\section{RESUlts AND Discussion}

The EEG data were collected under the cognitive tasks of studying the differences between the identification of Chinese and English sentences for Chinese students. Six male children, aging from 11 to 13 , were presented with visual stimulus of Chinese or English sentence. The sentence stimulus starts at $25 \mathrm{~ms}$, and then $26-250 \mathrm{~ms}$ were the information processing period of identifying the sentences. Subjects were requested to give, following the presentation of each stimulus, a reaction to press a button when the grammar of the sentence was identified as true or false. Our interest was focused on the dynamic synchronization with the front-right regions of the brain and the others. Multi-dimensional EEGs were collected via the 128-channel Geodesic EEG/ERP system with the sampling frequency of $250 \mathrm{~Hz}$, as shown in Fig. 4 . The channel 3 was selected as the reference for estimating the synchronizations with other 127 sites within the specified short period. In addition, we calculated a topographical synchronous mapping to exhibit the transient global coherence among the multichannel EEGs. Fig.5 and Fig.6 show the transient coherent topography of the synchronizations within the frequency band from 18 to $25 \mathrm{~Hz}$ between the reference and other 127 channels before and after justifying the given sentences. Three topographical mappings represent the average results for $1-25 \mathrm{~ms}, 26-150 \mathrm{~ms}$ and $151-250 \mathrm{~ms}$, respectively. Fig. 5 shows the results of before and during identifying the Chinese sentence, whereas Fig. 6 demonstrates the result when identifying the English sentence.

Based on the preliminary analysis, there exists a significant difference between first period $(1-25 \mathrm{~ms})$ and the second period (26-150ms) for processing the identification of both English and Chinese sentences. A lot of information exchanges significantly take place during the period of "sentence justification", while the synchronizations were small before identifying the sentences within 1-25ms. Moreover, it can be seen that the regions of information exchange when performing the Chinese sentence identification is on the middle of the brain, whereas the significant region of information exchange during English sentence justification is mainly on the heel of the brain. In the period of $26-150 \mathrm{~ms}$ for identifying the sentence, the synchronizations between different brain regions significantly increase. The similar results were true for the second period of $151-250 \mathrm{~ms}$ for identifying the sentences. Finally, by compared with the results between identifying the Chinese sentences and the English sentences, the preliminary results indicated that there were more synchronizations during justifying the Chinese sentences than English sentences for Chinese students.

\section{ConClusions}


A novel time-frequency coherence analysis based on wavelet analysis is proposed for investigating the time-varying synchronizations of EEGs between different brain regions for the cognitive task of identifying sentences in both Chinese and English. As a complex mother wavelet, Morlet wavelet was applied to derive the time-varying coherence analysis of multi-channel EEG signals. Wavelet coherence is defined that enables to investigate the synchronization and the information flow during Chinese and English sentence learning. For Chinese students, the different brain regions have different transient degrees of synchronization and information exchange patterns during processing the identification of Chinese and English sentence. The synchronization in time-frequency plane can be clearly identified with the cross wavelet magnitude spectra and the phase difference at the synchronized frequency.

The results exhibited that the wavelet coherence gives a better temporal and spectral resolution for time series analysis than traditional Fourier and AR model analysis. Different regions of the brain have different degree of the synchronization and the information exchange during processing the identification of a sentence. The results also indicate that the EEG signals recorded at one particular site may have been originated from different sources not directly beneath the skull surface at which the electrode is located. The time-varying synchronization between EEG signals from different sites provides us a new way to effectively track the changing procedure of the coherence and extract the neural information of EEG signals on how a learning process is in action. The proposed approach also proves the effectiveness and applicability for other non-stationary physiological signals.

\section{ACKNOWLEDGEMENT}

This work was supported in part by the National Natural Science Foundation of China (60271023), the Key Grant of Natural Science Foundation of Guangdong, China (021264) and the Key Grant of the Education Ministry of China (02110).

\section{REFERENCES}

[1] P. L. Nunez, R. Srinivasan, EEG coherence I.:statistics, reference electrode, vdume conduction, 1 aplacians. cortical imaging and interpretation at multiple scales. Electroen. And Clin Neurophysiol vol.103, pp.499-515, 1995.

[2] C. W. Anderson and E. A. Stolz, Multivariate autoregressive models for classification of spontaneous electroencephalogram during mental tasks. IEEE Trans Biomet Eng. Vol.45(3), pp.277-86, 1998.

[3] E. Moller and B. Schack, Instantaneous multivariate EEG coherence analysis by means of adaptive high-dimensional auto regressive models. $J$. of
Neuroscience Methods. Vol.105, pp.143-158, 2001.

[4] C. Andrew and G. Pfurscheller, Dependence of coherence measurements on EEG derivation type, Med. \& Biol. Eng. \& Comput., Vol.34, pp.232-238, 1996.

[5] B. Schack and P. Rappelsberger, Adaptive phase estimation and its application in EEG analysis of word processing, J. of Neuroscience Methods. Vol. 93, pp.49-59, 1999.

[6] B. Schack and G. Grieszbach, Dynamic cross spectral analysis of biological signals by means of biovariate ARMA processes with time dependent coefficients, Med. \& Bio. Eng. \& Com., Vol. 33, pp.605-610, 1995.

[7] B. Schack and W. Krause, Instantaneous Coherence as a Sensible Parameter for Considering Human Information Processing, IEEE Proceedings of ICPR'96, pp.45-49, 1996. [8] S. Weiss and P. Rappelsberger, EEG coherence within $13-18 \mathrm{~Hz}$ band as a correlate of a distinct lexical organization of concrete and abstract nouns in humans, Neuroscience Letters, Vol.209, pp.17-20, 1996.

[9] W. H. R. Miltner and C. Braun, Coherence of gamma-band EEG activity as a basis for associative learning, Nature, Vol.397, pp.434-435, 1999. 